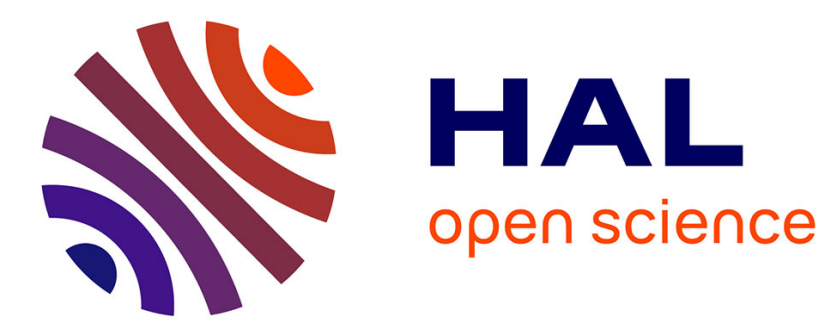

\title{
Apprendre à expliquer un texte philosophique à l'aide de la philosophie du cinéma \\ Hugo Clemot
}

\section{To cite this version:}

Hugo Clemot. Apprendre à expliquer un texte philosophique à l'aide de la philosophie du cinéma. Revue de l'enseignement philosophique, 2014, 65e année (2), pp.78-84. hal-03513086

\section{HAL Id: hal-03513086 \\ https://hal.science/hal-03513086}

Submitted on 5 Jan 2022

HAL is a multi-disciplinary open access archive for the deposit and dissemination of scientific research documents, whether they are published or not. The documents may come from teaching and research institutions in France or abroad, or from public or private research centers.
L'archive ouverte pluridisciplinaire HAL, est destinée au dépôt et à la diffusion de documents scientifiques de niveau recherche, publiés ou non, émanant des établissements d'enseignement et de recherche français ou étrangers, des laboratoires publics ou privés. 



\title{
III - TÉMOIGNAGES ET SUGGESTIONS
}

\author{
APPRENDRE À EXPLIQUER UN TEXTE PHILOSOPHIQUE
}

\author{
À L'AIDE DE LA PHILOSOPHIE DU CINÉMA
}

Hugo CLÉMOT

Lycée Paul-Louis Courier, Tours

Dans une série d'articles commencée en $1984^{1}$, le philosophe américain Noël Carroll a proposé un modèle « érotétique » du récit pour rendre compte de la « clôture narrative » (narrative closure) cinématographique. Cette conception soutient que l'impression de finalité ${ }^{2}$ que l'on ressent à la fin de la plupart des films est provoquée par le fait que les questions que le film a soulevées dans l'esprit du spectateur trouvent une réponse. Le plaisir pris à suivre la plupart des récits filmiques s'expliquerait donc en partie par un besoin naturel de trouver des réponses aux questions qu'ils nous font nous poser. Cet article entend montrer quel profit le professeur de philosophie peut tirer du modèle érotétique pour aider ses élèves à approcher les textes philosophiques en leur proposant d'abord de les concevoir comme étant structurés par un jeu de questions et de réponses que le lecteur doit retrouver pour mettre en relation les parties du texte en un tout cohérent. L'analogie filmique permet également de prendre conscience de cé que l'auteur d'un texte philosophique écrit toujours en prêtant attention à la voix de ses lecteurs, à commencer par le premier d'entre eux, c'est-à-dire lui-même. La conjonction du modèle érotétique et de l'idée qu'un texte philosophique est toujours traversé par plusieurs voix conduit à justifier la pratique d'une mise en dialogue de textes philoso-

\footnotetext{
1. Noël Carroll, « Toward a Theory of Film Suspense », Persistence of Vision. The Journal of the Film Faculty of the City University of New York, $\mathrm{n}^{\circ} 81$, 1984, p. 65-89; repr. in Theorizing the Moving Image, New York, Cambridge University Press, 1996, p. 94-117; « The Power of the Movies », Daedalus, vol. 1, n 14, 4, automne 1985, p. 79 104; repr. in Theorizing the moving picture, ibidem, p. 78-93; «Narrative closure », Philosophical Studies; An International Journal for Philosophy in the Analytic Tradition, vol. 135, $\mathrm{n}^{\circ} 1$, aotit 2007, p. 1-15; The Philosophy of Moving Pictures, Malden, MA, Blackwell,2008, chap. 5, section 2 « Cinematic Narration », p. 133-146; « Narrative closure ", Paisley Livingston, Carl Plantinga (ed.), The Routledge Companion to Philosophy and Film, Londres, Routledge, 2009, chap. 19, p. 207-216; Art in three dimensions, chap. 17, « Narrative closure », New York, Oxford University Press, 2010, p. 355-372.

2. Carroll, « Narrative closure », Philosophical Studies, abstract, art. cit., p. 1.
} 
phiques qui permet à la fois leur analyse et prépare la description fine des opérations textuelles de l'auteur.

\section{LE MODÈLE ERROTÉTIQUE D'ANALYSE DU RÉCIT}

Partant d'une conception causaliste du récit selon laquelle un discours est un récit si et seulement s'il décrit au moins deux événements temporellement et causalement reliés l'un à l'autre ${ }^{3}$, le philosophe américain Noël Carroll introduit un modèle érotétique en vue de répondre à l'objection suivante: si le récit compte au titre de ses éléments nécessaires une connexion narrative ${ }^{4}$, c'est-à-dire une relation causale entre événements, comment rendre compte de l'impression de clôture que l'on ressent quand toutes les questions posées par le récit ont trouvé une réponse? Quelle est l'origine de ce sentiment?

Selon le modèle érotétique de la relation interrogative utilisé par Poudovkine ${ }^{5}$, le sentiment de clôture tiendrait à la satisfaction d'un besoin naturel de trouver des réponses à nos questions, besoin sur lequel les narrateurs s'appuieraient pour relater les événements liés par des relations caụsales en construisant leur récit pour susciter des questions que celui qui suit le récit doit se poser, plụs ou moins consciemment ${ }^{6}$, quitte à manipuler la temporalité, en usant du flash-back ou du flashforward notamment, en vue de fournir les éléments nécessaires pour que les questions se posent et que les réponses soient données ${ }^{7}$.

S'il fait référence à Poudovkine, Carroll remarque aussi que l'on trouve chez Hume un principe narratif équivalent dans la remarque suivante, issue de l'essai De la tragédie ${ }^{8}$ :

Si vous aviez l'intention d'émouvoir fortement une personne par le récit d'un événement, la meilleure méthode pour accroître son effet serait de retarder avec art le moment de l'en informer, et d'exciter d'aborḍ sa curiosité et son impatience avant de lui livrer le secret ${ }^{9}$.

Le « principe de Hume ${ }^{10}$ » serait donc de présenter au spectateur une chaîne d'événements dont il serait curieux de connaître le résultat, mais en retardant le moment de lui faire connaître ce résultat. Le « secret » ne serait qu'une question ou, si l'on généralise ce principe à un récit entier, un ensemble de questions auxquelles le spectateur attend que l'on réponde. La clôture du récit serait assurée quand toutes les questions qui auront suscité la curiosité du spectateur auront trouvé une réponse. Cela ressemble en effet au modèle érotétique: un récit narratif clos est un réseau de questions et de réponses, où toutes les questions soulevées trouvent au final une réponse.

Pour illustrer la vérité de ce principe et valider le modèle érotétique d'analyse du récit, Carroll renvoie d'abord à l'expérience, aisée à réaliser, qui consiste à interrompre une projection filmique avant la fin: les réactions de protestation parfois vives des spec-

3. Carroll, "Narrative closure », The Routledge Companion to Philosophy and Film, op cit., p. 207; Art in three dimensions, op. cit., p: 367.

4. Carroll, « On Narrative Connection », Beyond Aesthetics, Cambridge, Cambridge University Press, 2001, p. 127; Art in three dimensions, op. cit., p. 367.

5. Vsevolod I. Poudovkine, Film Technique in Film Technique and Film Acting, New York, Grove Press, Inc., 1958, p. $73 ; 77$.

6. Carroll, «Toward a Theory of Film Suspense », Theorizing the Moving Picture, op. cit., p. 96.

7. Carroll, « Narrative closure », The Routledge Companion to Philosophy and Film; op. cit., p. 213 ; Art in three dimensions, op. cit., p. 366 .

8. Voir Hume, De la tragédie (1757), dans Hume, Essais esthétiques, trad. R. Bouveresse, Paris, GF-Flammarion, 2000, p. 111-121.

9. Hume, De la tragédie, op. cit., trad. modifiée, p. 117.

10. Carroll, The Philosophy of Moving Pictures, op. cit., p. 135. 
tateurs semblent en effet témoigner du besoin pressant de répoṇse aux questions que le récit nous a fait nous poser.

Il cite aussi plusieurs auteurs qui semblent retrouver ce principe ${ }^{11}$, avant de procéder à l'analyse rapide de plusieurs récits susceptibles de provoquer cette « impression de finalité, phénoménologiquement parlant ${ }^{12}$ », qu'il appelle des « récits érotétiques ${ }^{13}$ ».

Par exemple, OEdipe roi s'ouvre sur l'annonce faite à GEdipe de ce qu'« une plaie tombée du ciel embrase la cité, [...] la Peste matudite ${ }^{14} »$. Cette annonce ne pourrait pas ne pas soulever la question de savoir si l'épidémie s'arrêtera. Lorsque l'on apprend ensuite que la peste s'arrêtera si le meurtrier de Laïos est puni, on en vient naturellement à se demander qui a tué Laïos et on ne tarde guère à s'interroger sur l'identité réelle d'CEdipe. Le récit trouverait sa clôture au moment où CEdipe est puni après avoir compris qui iI est réellement et donc ce qu'il a fait, e'est-à-dire au moment où le lecteur trouve les réponses aux questions précédentes. En effet, la peste peut s'arrêter puisque les conditions pour qu'elle disparaisse sont réunies.

Les questions dont la clôture narrative dépend n'apparaissent donc pas au hasard: elles sont intentionnellement provoquées par l'auteur du récit qui emploie plusieurs scènes et séquences pour que les conditions de leưr position soient elles-mêmes réunies. Ainsi, l'analyse érotếtique du récit comme un ensemble de scènes ou de séquences structurées par un réseau de questions et de réponses nous permet de distinguer plusieurs fonctions, plusieurs rôles que les scènes ou les séquences d'un récít peuvent jouter. Aux scènes d'exposition d'un film dont la fonction est de répondre aux premières questions que soulève généralement tout discours qui prétend être un récit ( où l'action a-t-elle lieu, quand, qui sont ces gens et quels sont leurs liens, que veulent-ils, avec ou contre qui sont-ils, pourquoi se comportent-ils ainsi, etc. ${ }^{15}$ »), il faut ajouter celles qui suscitent des questions, celles qui cherchent à y répondre, celles qui visent à soutenir (sustain) des questions antérieures, celles encore qui ne répondent qu'incomplètement ou qui répondent en posant d'autres questions ${ }^{16}$.

Les questions soulevées par le récit ne se distinguent pas seulement selon leurs fonctions, mais encore selon leur importance pour la structure du récit. Ainsi, on doit distinguer les « macroquestions directrices » des « microquestions », les secondes portant sur les moyens adoptés par le protagoniste pour atteindre ses fins qui sont définies par les réponses aux premières. Il y a une relation hiérarchique entre les questions posées par un récit en cours ${ }^{17}$, relation qui définit la structure générale du récit.

\section{LE MODĖLE ÉROTÉTIQUE APPLIQUÉ À L'EXERCICE D'EXPLICATION DE TEXTE}

L'enseignant de philosophie, confronté à la difficulté qu'il y a à permettre à̀ ses élèves d'éviter de se contenter de paraphraser les textes philosophiques qu'ils ont à expliquer, c'est-à-dire à dire en général moins bien ce que l'auteur a déjà très bien écrit,

11. Eudora Welty, Edgar Allen Poe, E. M. Forster ou Roland Barthes.

12. Carroll;, « Narrative closure », Philosophical Studies, abstract, art. cit., p. 5.

13. Ibid.

14. Sophocle, EEdipe roi dans Théâtre complet, Paris, GF Flammarion, trad. Robert Pignarre, 1964, p. 106.

15. Carroll; " Narrative closure », Philosophical Studies, abstract, art. cit., p. 5.

16: Ibid. Voir l'exposé des six ou sept types de scènes (scènes d’exp̣osition, dè question, de réponse, de soutien, de réponse incomplète, de réponse incornplète et āmbiguë et de réponse qui soulève une question) đanș Carroll, « Toward a Theory of Suspense » (1984), in Theorizing the Moving Image, op. cit., p. 98 et note 13, p. 114.

17. Carroll; « Namative closure », Philosophical Studies, abstract, art. cit, p: 6: « Les microquestions sont généralement subordonnées aux macroquestions directrices. Ea clôture est obtenue quandị toutes les macroquestions directrices et toutes les microquestions pertinentes pour résoudrẹ les macroquestions aụront trouvé une répoinse: » 
aura peut-être déjà saisi l'intérêt de la théorie de Carroll : si l'analyse érotétique du récit comme un ensemble de scènes ou de séquences structurées par un réseau de questions et de réponses nous permet de distinguer plusieurs fonctions, plusieurs rôles que les scènes ou les séquences d'un récit peuvent jouer, peut-être l'analyse érotétique d'un texte philosophique que l'on concevrait comme un ensemble de concepts, de jugements et de raisonnements structurés par un réseau de questions et de réponses hiérarchisées pourrait-elle permettre aux élèves de distinguer plusieurs fonctions, plusieurs rôles que les concepts, jugements et raisonnements d'un texte philosophique peuvent jouer?

La mise en place d'une séquence autour de ce principe est relativement aisée dans la mesure où il suffit de sélectionner un extrait de l'une des séries télévisées (ou de l'un des films) les plus appréciées des élèves pour leur faire découvrir en pratique et d'euxmêmes cette deuxième dimension constitutive, en général inaperçue, de l'œuvre qu'ils apprécient, en enrichissant ainsi leur expérience de spectateur. On peut ensuite les inviter à procéder de la même façon pour un texte philosophique et justifier àinsi une démarche bien connue de tous les professeurs.

Par exemple, le fameux texte de la deuxième préface de la Critique de la raison pure qui commence par «Lorsque Galilée fit descendre sur un plan incliné ses boules... » et finit par « [...] mais comme un juge en charge, qui force les témoins à répondre aux questions qu'il leur pose ${ }^{18}$. » sera analysé selon un plan découvert à partir du jeu de questions et de réponses qui structurent le texte. Dans le cadre d'une formation disciplinaire qu'il animait naguère à l'IUFM de Nantes, Jean Kérinvel présentait les choses ainsi:

D'où tirer un enseignement sur la manière rationnelle de connaître? Des démarches de savants éminents.

2. Quelle leçon en tirer? Qu'en ont-ils tiré en ce qui concerne le pouvoir de la raison? Que pouvons-nous plus généralement en tirer pour déterminer l'attitude rationnelle? Sous quelle forme cette initiative va-t-elle se référer aux phénomènes extérieurs?

3. Pourquoi une telle règle? N'est-ce pas arbitraire? Voire opposé au principe d'une connaissance qui doit se conformer à son objet? Quelle est la nécessité de cette attitude?

4. Si la raison est liante et nécessitante, quelle forme déterminée va prendre cette initiative liante?.(Pourquoi cette redondance apparente?) Comment interroger la nature? « À deux mains. » Comment expérimente-t-on?

5. Comment mieux comprendre cette approche complexe de la nature? $\grave{A}$ quelle attitude familière s'oppose-t-elle? À quelle activité familière peut-elle être au contraire assimilée?

La description des opérations textuelles n'en sera alors rendue que plus aisée. Il sera également possible de remarquer que l'attitude rationnelle finalement prônée par Kant vis-à-vis de la nature, celle du juge qui ne se contente pas de répéter passivement ce que le maître lui a appris, mais qui cherche à déterminer activement quelles questions poser pour trouver les bonnes réponses, est exactement celle que l'on doit adopter pour comprendre et expliquer un texte philosophique: ne pas se contenter de répéter ce que l'auteur a dit, mais déterminer les questions que le discours tenu peut susciter, et chercher dans le texte des réponses.

\section{APPRENDRE À ÉCOUTER LES VOIX QUI TRAVERSENT UN TEXTE PHILOSOPHHQUE PAR LA MISE EN DIALOGUE}

Le modèle érotétique proposé par Noël Carroll ne permet pas seulement de rendre les élèves attentifs aux opérations textuelles de l'auteur, c'est-à-dire à tout ce que

18. Kant, Critique de la raison pure, B XIII-BXIV, préface de la seconde édition [1787], trad. A. J.-L. Delamarre, F. Marty et J. Barni, Paris, Gallimard, « Folio essais »-1995, p. 43-44. 
l'auteur fait pour susciter des questions dans l'esprit du lecteur et y répondre. En effet, après avoir invité l'élève à se mettre à la place du spectateur, c'est-à-dire à analyser les questions et les réponses qu'il est conduit plus ou moins consciemment à se poser, l'enseignant peut ensuite l'inciter à prendre conscience de ce que l'auteur d'un texte l'écrit toujours en anticipant les questions et la saisie des réponses de ses lecteurs. Ce peut d'ailleurs être l'occasion d'avancer certains éléments d'analyse du concept de " pensée " en justifiant l'idée, qu'on lit chez Peirce en note de bas de page de Platon ${ }^{19}$, selon laquelle « [1]a pensée procède sous la forme d'un dialogue - un dialogue entre différents stades de l'ego. [...] Toute pensée est dialogique dans sa forme. Le soi en un instant donné appelle le soi plus profond pour son assentiment [...] ${ }^{20}$. Sans entrer dans les détails, ni préciser les différences de perspectives des deux philosophes, le commentaire peut être illustré par une séquence de la série bien connue des élèveś, Dr House, où le personnage principal, souffrant d'hallucinations, dialogue littéralement avec un autre lui-même en en étant conscient ${ }^{21}$ et conduire à remarquer que l'auteur est toujours son premier lecteur et donc en cela toujours un deuxième auteur, une deuxième voix en puissance.

Autrement dit, il n'est pas exagéré de penser qu'il y a toujours plusieurs voix, quii ne sont d'ailleurs pas toujours tues, dont l'expression détermine le cours du texte. Il n'est donc pas illégitime de suggérer aux élèves de repérer à la fois le jeu des quiestions et des réponses qui structurent le texte et les voix qui le travęrsent par la pratique de la mise en dialogue de textes philosophiques. En effet, cette forme dialogique permet à la fois l'analyse du texte et prépare la description fine des opérations textuelles de l'auteur. Par exemple, lorsque le lauréat du Grand Prix de philosophie de l'Académie française, Charles Larmore, analyse la démarche qui mène Descartes, dans ses Méditations métaphysiques, à son fameux cogito, il ne propose pas autre chose qu'une-mise en dialogue qui met en évidence l'existence de plusieurs voix dans son texte ${ }^{22}$. De même, un grand nombre de commentateurs des Recherches philosophiques de Wittgenstein reconnaissent aujourd'hui la vérité de ce que Stanley Cavell a été l'un des premiers à mettre en évidence, à savoir qu'on ne doit pas lire ce livre comme un monologue, ni même seulement comme un dialogue, mais comme un échange entre plusieurs voix qui s'accordent dans le langage malgré leurs désaccords, sans que cet accord soit certain, ni pérenne ${ }^{23}$.

Pour ne donner qu'un exemple, voici une tentative de mise en dialogue qui prépare l'explication d'un texte dense d'un point de vue argumentatif, le passage fameux du Traité de la réforme de l'entendement où Spinoza critique explicitement les sceptiques ${ }^{24}$ :

[Les sceptiques] n'ont même pas conscience d'eux-mêmes [1]; s'ils affirment quelque chose ou doutent de quelque chose, ils ne savent pas qu'ils affirment ou qu'ils doutent [2]; ils disent qu'ils ne savent rien [3], et cela même qu'ils ne savent rien, ils déclarent l'ignorer [4]; encore ne le disent-i]s pas sans restriction, car ils craignent de s'avouer existants, alors qu'ils ne savent rien [5], si bien qu'il leur faut enfin garder le silence pour être sûrs de ne rien admettre qui ait senteur de vérité [6]. Il faut, en définitive, s’abstenir de

19. Platon, Théétète, 189e-190a, trad. E. Chambry, Paris, GF Flammarion, 1967, p. 136.

20. Charles Sanders Peirce, Collected Papers, publiés par C. Hartshorne et P. Weiss, Harvard University Press, $1931-1958,4.6 ; 6.338$

21 Voir Dr House (série TV, David Shore, saison 2, épisode 24, 2006).

22. Voir Charles Larmore, « La structure dialogique de la première Méditation », Philosophie, 65, 2000, p. 55-72.

23. Voir Stanley Cavell, Les Voix de la raison. Wittgenstein, le scepticisme, la moralité et la tragédie, trad, fr.

S. Laugier et N. Balso, Paris, Seuil, 2012 (1996); sur l'importance de cette idée, voir David Stern, Wittgenstein's Philosophical Investigations: an introduction, Cambridge, Cambridge University Press, 2004, p. 21-28.

24. Spinoza, Traité de la Réforme de l'Entendement, \& 31, trad. Charles Appuhn, Paris, GF Flammarion, 1964, p. 194-195. 
parler de sciences avec eux [7]: (car pour ce qui concerne l'usage de la vie et de la société, la nécessité les oblige à admettre leur propre existence, à chercher ce qui leur est utile, à affirmer et à nier sous serment bien des choses [8]). Leur prouve-t-on quelque chose, en effet, ils ne savent si l'argumentation est probante ou défectueuse. S'ils nient, concèdent, oụ opposent une objection, ils ne savent qu'ils nient, concèdent, ou opposent une objection [9]. Il faut donc les considérer comme des automates entièrement privés de pensée [10].

Le dialogue est rédigé de façon à préparer la description de la fonction de chaque concept, jugement et raisonnement présent dans chacun des dix passages du texte ou constitué à partir d'eux:

SPINOZA: «Vous n'avez même pas conscience de vous-même. » [Première affirmation de Spinoza].

SCEPTIQUE: Pourquoi dites-vous cela?

SPINOZA: Je vais vous l'expliquer. Vous dites que l'on ne peut rien savoir avec certitude, n'est-ce pas? [1]

SCEPTIQUE : Je ne sais pas si je l'affirme ou si j'en doute puisqu'on ne peut rien savoir avec certitude.

SPINOZA: Mais que savez-vous alors? [2]

SCEPTIQUE: Je ne sais rien.

SPINOZA: Mais reconnaissez que vous savez, comme Socrate, au moins une chose: que vous ne savez rien. [3]

SCEPTIQUE: Non, je l'ignore puisque je ne sais pas si c'est certain et cette ignorance elle-mêmè est inçertaine.

SPINOzi: Mais que vous sachiez quelque chose ou que vous l'ignoriez, vous savez au moins que vous existez puisque vous ne pourriez pas douter sans exister. [4]

SCEPTIQUE: Je ne sais pas avec certitude si j'existe.

SPINOZA [tire trois conséquences logiques du discours du sceptique] : Puisque vous ne savez jamais rien, pas même que vous existez, vous n'avez donc pas conscience de vous-même puisque avoir conscience, c'est savoir ce qu'on fait, pense ou ressent. [Première conséquence: où l'on vérifie la première affirmation de Spinoza]. [5]

Vous feriez donc mieux de vous taire, car chaque phrase que vous formuleriez risquerait de témoigner d'un savoir que vous prétendez ne pas avoir. [Deuxième conséquence: où l'on déduit de tout ce qui précède que le scepticisme conduit logiquement au silence]. [6]

D'ailleurs, je vais mettre un tèrme à notre discusssion, car il est impossible de chercher la vérité avec vous. [Troisième conséquence: où l'on comprend que l'on n'a pas à écouter ce que les sceptiques ne devraient de toute façon pas dire]:

[SCEPTIQUE: pourquoi dites-vous cela ?] [7]

SPINOZA [emploie deux nouveaux arguments pour justifier l'idée précédente] : Je viens" de vous expliquér pourquoi vous feriez mieux de vous taire. En outre, vous n'êtes pas un interlocuteur fiable, car vous ne vous comportez pas en sceptique quand vous ne parlez pas de sciences. Je remarque en effet que votre conduite dans la vie témoigne d'un certain nombre de savoirs et de savoir-faire dont vous ne doutez pas: par exemple, vous ne vous comportez pas en sceptique quand votre vie est en danger, quand il vous faut faire quelque chose pour vous nourrir ou quand on vous demande de promettre sous peine de mort. [Premier argument]. [8]

Enfin, la discussion scientifique suppose la maîtrise d'un certain nombre de capacités logiques : il faut savoir distinguer les argumentations probantes des preuves défectueuses, savoir si l'on veut contester, accepter ce qui est dit ou soulever une 
objection. Or, puisque vous affirmez ne rien savoir, vous n'êtes pas un interlocuteur compétent. [Deuxième argument]. [9]

Vous pouvez bien agir au quotidien comme un homme et émettre des sons qui s'apparentent à la voix humaine, puisque vous refuserez toujours de reconnaître que vous savez ce que vous venez dire, je ne vous considère que comme un automate entièrement privé de pensée. [Des deux derniers arguments, Spinoza déduit donc de nouveau qu'il ne faut pas discuter avec les sceptiques si l'on cherche la vérité, mais les tenir comme des « automates entièrement privés de pensée », ce qui vérifie la première affirmation du texte et boucle l'argumentation comme la fin du film vient clôturer le récit]. [10]

\section{CONCLUSION}

Il n'est pas sûr que le sceptique qui sommeille en chacun des lecteurs se laisse aussi facilement réduire au silence et accepte qu'on le tienne aussi violemment pour un interlocuteur incompétent, de même qu'il n'est pas sûr que la démarche pédagogique ici décrite et justifiée recueille le plein assentiment de ses lecteurs, y compris de l'auteur lui-même. Dans le contexte qui caractérise aujourd'hui les conditions d'enseignement de la philosophie dans le secondaire, il devient en effet de plus en plus difficile de supporter les discours de ceux qui prétendent savoir comment il faut enseigner. Plus qu'en d'autres matières encore, une certaine dose de scepticisme semble nécessaire qui permette l'expérimentation, la mise en pratique, en un mot qui encourage à faire l'expérience par soi-même plutôt que de décider a priori que les choses doivent ou ne doivent pas se passer ainsi. De plus, enseigner la philosophie à l'aide du cinéma est une pratique ancienne et qui n'a plus besoin aujourd'hui d'être justifiée. Cependant, l'article aura voulu suggérer, à partir d'un exemple, que les professeurs de philosøphie pourraient enrichir leur pratique pédagogique en s'instruisant de la philosophie dụ cinéma. 